\title{
ÉTUDIER L'HYBRIDATION ORGANISATIONNELLE PAR LE PRISME DES PUBLICS : LES PUBLICS COMME PIERRE ANGULAIRE DES TENSIONS QUI ANIMENT UNE ASSOCIATION DE SOLIDARITÉ INTERNATIONALE ${ }^{1}$
}

\author{
Sandrine RoginSKY ${ }^{2}$ et Christel CHRISTOPHE ${ }^{3}$
}

Comment les acteurs d'une organisation se représentent les publics que ses activités visent ? Comment cette activité de représentation, ou de figuration, nous renseigne-elle sur la nature hybride de l'organisation et sur les tensions qui l'animent ? Ces questions indiquent une voie originale pour l'analyse des logiques organisationnelles d'hybridation qui émanent de différents mondes sociaux. Celle-ci mobilise une approche pragmatique et constructiviste de la communication, envisagée comme constitutive de l'organisation. Grâce à l'analyse discursive des procédés rhétoriques mobilisés par les salariés d'une association de solidarité internationale pour figurer leurs publics, nous restituons les traces des logiques organisationnelles en tension. Deux usages dominants de la figure du public émergent alors des processus de figuration, amenant avec eux des référentiels d'évaluation divergents : d'une part une approche politique et d'autre part une approche gestionnaire de l'organisation. Nous montrons

1 Les auteures tiennent à remercier les personnes qui ont évalué cet article pour leurs remarques riches et pertinentes qui ont permis d'améliorer et préciser la thèse qu'elles développent.

2 Sandrine Roginsky est professeure à la Faculté des sciences économiques, sociales, politiques et de communication à l'Université catholique de Louvain.

3 Christel Christophe est assistante à la Faculté des sciences économiques, sociales, politiques et de communication à l'Université catholique de Louvain.

Recherches en communication, $\mathrm{n}^{\circ} 47$ - Article publié le 19/12/2018 
ainsi l'apport d'une approche par les discours sur les publics pour analyser l'hybridation organisationnelle.

Pourquoi étudier l'hybridation organisationnelle ${ }^{4}$ par le prisme des publics alors que « la question des publics est sans doute l'une des plus controversées parmi toutes celles que les sciences de l'information et de la communication se proposent de traiter » (Esquenazi, 2006, p. 11) ? C'est à cette question que le présent article souhaite répondre. Derrière l'abstraction d'un terme flou, qui renvoie à différents univers de discours - puisque, comme le rappelle Dayan (1992, p. 156), « la notion de public est construite de toutes pièces par les discours portés sur lui »-se trouve néanmoins un objet « que l'on peut indiquer [ou auquel] on peut faire référence » (Blumer, 1969, p. 10), mobilisé par des acteurs d'une organisation qui cherchent à communiquer auprès desdits publics. La question des publics est en effet une question centrale, puisque « toute action est un acte de communication qui s'adresse à un auditoire » (Abbott, 2016, p. 89) ; par conséquent, toute action entreprise par une organisation s'adresse d'une manière ou d'une autre à un ou des publics. Ainsi, c'est aux publics figurés dans les discours des acteurs que nous nous intéressons, en explorant l'agentivité des représentations véhiculées à travers ces discours. Nous proposons de retracer les figures du public dans les discours et cherchons à saisir leur variété. Derrière l'acception du terme «public », des figures antagonistes se confrontent et transportent des valeurs et des principes qui le sont tout autant. De fait, elles témoignent des différentes logiques organisationnelles sous-jacentes à l'hybridité organisationnelle. Pour le dire autrement, les publics figurés de multiples manières participent à la configuration de l'organisation. De ces manières de figurer émergent des figures antagonistes du public qui rendent ainsi visible l'hybridité organisationnelle dont ils sont à la fois cause et conséquence. Par le prisme du public, c'est donc l'organisation qui est interrogée.

4 L'hybridation organisationnelle est ici définie comme l'interaction et l'entrelacement de différentes logiques organisationnelles qui produisent un assemblage hétérogène. 
L'article se veut une contribution aux réflexions portées par les tenants de l'approche constitutive des organisations (CCO). En s'intéressant à la manière dont les publics sont rendus visibles par des figures divergentes (dont l'agentivité participe à construire l'organisation hybride), l'article souhaite démontrer que les tensions construites par les figures divergentes des publics constituent l'organisation. Ce faisant, il explore les processus d'émergence des figures construites et négociées dans le discours et montre que ceux-ci présentent des degrés d'objectivation sociale variables. La notion d'objectivation sociale nous semble clé pour saisir les processus de figuration, desquels émergent les figures ici des publics - car elle permet d'appréhender le degré d'intégration collective des significations. Ce qui amène au dernier élément contributif de cet article qui vise à intégrer la notion de réflexivité des acteurs dans une perspective $\mathrm{CCO}$, notamment en incluant dans l'analyse différents types de conversation et en explorant la notion de monde social qui nous semble apporter une lumière utile à l'analyse des interactions dans le cadre des approches CCO. Une telle démarche permet de dépasser " l'aporie du focus sur [une] seule interaction » (Jojczyk et al., 2016, para. 9). Nous souhaitons ainsi continuer la réflexion initiée par Jojczyk et al. (2016) qui notent qu'en se focalisant sur une interaction, le chercheur n'a pas accès à « des éléments pourtant cruciaux permettant de faire sens de l'interaction » (ibid.).

L'article déroule plusieurs étapes : il s'agit d'abord de mettre en lumière le lien entre les notions de public et d'organisation dans une perspective $\mathrm{CCO}$ puis de définir les concepts de figure et d'agentivité ; il s'agit ensuite de présenter et justifier les conversations qui sont intégrées dans l'analyse car elles nous permettent d'explorer les processus de figuration tels qu'ils se déploient dans les conversations entre les acteurs. Il est alors possible de saisir comment les figures du public émergent dans les conversations et entrent en tensions et comment les tensions constituent l'organisation. Enfin, l'article conclut par des propositions d'ordre méthodologique et théorique pour explorer les processus de figuration, en tenant compte tout à la fois de la réflexivité des acteurs et de celle du chercheur. 


\section{Quand les publics rencontrent l'organisation : une contribution à la $\mathrm{CCO}$}

La convergence entre les notions de public et d'organisation ne va pas de soi : la première est analysée par les études de réception, dans la tradition de la sociologie des médias, et par la sociologie de la culture. La seconde est notamment approchée par le courant de la CCO. Et pourtant des zones de convergence existent. D'ailleurs, remarquons que la question du public est centrale dans la réflexion développée par Dewey, philosophe du pragmatisme américain qui a influencé les chercheurs qui se situent dans le courant constructiviste, parmi lesquels des tenants de la CCO. Dewey (1927/2010) s'interroge sur ce qu'il nomme «l'éclipse du public » et sur sa fonction. Pour Kruckeberg et Vujnovic (2010), c'est à ce philosophe qu'on doit le concept du public, à la fois dans son usage singulier et pluriel. Esquenazi (2009) considère le concept de public comme un objet frontière. Assogba et ses collègues (2015, para. 2) notent ainsi le « brouillage des frontières entre les concepts de public, de communauté, de collectif d'usagers ou encore d'audience ». Ce flou terminologique nous amène à aborder la question des publics en nous intéressant aux principes de jugement et d'appréciation employés pour qualifier ces collectifs. Nous nous intéressons ici au «travail de représentation » (Denis, 2008) par lequel l'absence physique des publics est rééquilibrée par des convocations régulières de ceux-ci, notamment dans les activités conversationnelles des acteurs.

Ce travail de représentation, ou de figuration, consiste donc à rendre présents les publics dans les activités de l'organisation. Dès lors, c'est bien en tant qu'objet, entendu comme « toute chose que l'on peut indiquer ou à quoi l'on peut faire référence » (Blumer, 1969 , p. 10), tel que figuré et communiqué par les acteurs, que nous analysons la performativité des publics dans la vie de l'organisation. On voit ici la pertinence d'intégrer le concept de public dans l'agenda de recherche du courant de la CCO. Ce qui nous amène à porter notre regard sur les figures du public qui émergent des discours. Si pour Meunier « connaître quelque chose, c'est avoir une représentation de ce quelque chose » (Meunier, 2003, 
p. 103), Cooren et Martine (2016, para. 14) nous rappellent quant à eux que « si quelque chose ou quelqu'un existe, c'est bien toujours qu'il se matérialise ou se concrétise, d'une manière ou d'une autre ». Connaître, matérialiser dans le discours, se représenter, sont autant d'activités qui permettent de rendre compte de cette modalité d'existence des publics.

\section{L'agentivité des figures du public et les processus de figuration}

Nous définissons ici la figure comme la conceptualisation du public tel que rendu présent dans le discours. Le public devient une notion qui dispose d'une agentivité. Le concept de l'agentivité s'inscrit dans l'approche CCO et déroule quatre principes (Baudry, 2016, p. 46) dont nous retenons ici le premier qui « met en évidence le caractère hétérogène des agents et la dimension hybride des interactions » et le second qui montre « les capacités de ces agents à agir sur et/ou à faire agir ». À la suite de Latour (2005), nous proposons d'appréhender les représentations des publics comme des figures (idéomorphiques) qui, bien que ne disposant pas de la matérialité corporelle propre à l'acteur humain, agissent dans les discours comme des véhicules qui nous offrent des explications sur les forces contraignant les logiques organisationnelles. Car, comme l'indique Cooren (2010a), des êtres apparemment plus abstraits, comme ici le public, ne manquent pas d'agir sur notre vie quotidienne. Ils le font par l'intermédiaire de « figures à ontologie variable [qui] s'expriment et s'incarnent dans l'interaction donnant du poids (et donc de l'autorité) à ce qui est mis de l'avant par les interlocuteurs humains » (Cooren, 2010b, para. 4).

Les figures des publics participent ainsi à configurer l'organisation et grâce à leur portée significative " s'incorporent dans les pratiques et les usages pour leur donner forme et les orienter » (Quéré, 2003, p. 114). Autrement dit, pour le dire plus simplement, les publics animés par des figures font faire des choses aux membres d'une organisation alors même qu'ils parlent en leur nom. Pour Jolivet et Vasquez (2011, para. 15), « toute figure est le résultat d'une configuration - un ensemble de valeurs et de principes qui 
s'imbriquent pour lui donner corps ». Les figures incarnées dans le discours transigent en effet des valeurs (Gagné, 2011). Mais que se passe-t-il quand celles-ci s'opposent, alors même qu'elles sont censées donner corps à un même objet ? Nous suivons ici Jolivet et Vasquez pour qui « la mise en scène de figures multiples - textuelles, humaines, architecturales, artefactuelles - dans des textes (écrits ou oraux) rend compte du mode d'existence pluriel de l'organisation, qui doit alors être conçu comme étant le résultat d'une configuration d'agentivités hybrides » (2011, para. 1). Ainsi, la variété des figures n'est finalement que le pendant de l'hybridité organisationnelle. On saisit alors pourquoi derrière l'acception du terme " public », différentes figures sont en compétition. Chaque figure propose une vision et des qualifications différentes, parfois contradictoires. Pour Cooren (2013), les tensions organisationnelles sont justement constituées par ces figures divergentes. Or, ce sont ces tensions, mises en acte dans les interactions, qui construisent la réalité organisationnelle. Ces tensions sont non seulement inévitables parce qu'elles sont inhérentes à toute forme organisationnelle (Trethewey et Ashcraft, 2004, p. 82), mais surtout elles sont animées par des figures qu'elles animent en retour (Cooren, 2010a). Ce faisant, les tensions sont constitutives de l'organisation.

Par ailleurs, les figures n'émergent pas de nulle part. Elles sont le fruit d'un travail de figuration. La figuration est « ce qui agit » et qui « revêt toujours, dans le compte rendu qui en est fait, une certaine consistance, une apparence qui lui donne une certaine forme et des contours, aussi vagues soient-ils » (Latour, 2005, p. 77). Il nous semble par conséquent important de prendre en compte les manières de figurer le public, les « régimes représentationnels » pour reprendre le vocabulaire de Denis (2008, p. 161), à partir desquelles les figures émergent. Autrement dit, il s'agit de comprendre pourquoi et comment une figure émerge, en ne nous limitant donc pas à la seule prise en compte des figures dans leur capacité à être animées tout en animant la conversation (Cooren, 2010a) mais en prenant également en compte les manières de faire apparaître les figures. La question à laquelle nous tentons ainsi de répondre pourrait se poser comme suit : comment s'y prend-on 
pour se figurer le public ? Cette étape est cruciale dès lors qu'il s'agit de se représenter le ou les publics auxquels on s'adresse.

\section{Trianguler différents types de « conversations »}

Nous nous arrêtons sur le cas spécifique d'une association belge de coopération au développement. Cette association de solidarité internationale déploie deux axes d'activité : le soutien à l'agriculture paysanne au Sud, la sensibilisation et la mobilisation au Nord. L'activité de sensibilisation conduit l'association à produire de l'information (Errecart, 2013 ; Carion, 2010) et à la diffuser auprès d'individus catégorisés en publics. Les publics jouent ici un rôle clé, puisque les actions menées sont pensées pour eux. Raison pour laquelle l'organisation en question nous a sollicitées pour mener une recherche sur les habitudes informationnelles de ses publics. À cette occasion, différents moments de conversation ont été organisés, que nous étudions ici.

Dans l'approche " texte-conversation " de la $\mathrm{CCO}$, une conversation est envisagée comme un événement emblématique d'un processus constant de redéfinition qui va mobiliser l'objet (le texte) discuté, en l'occurrence ici la compréhension des publics. Nos données sont des conversations qui s'apparentent davantage à des discussions réflexives, à des méta-conversations (Robichaud et al., 2004) - des conversations sur l'organisation - lors desquelles nous pouvons, sans risque d'extrapolation, identifier comment les participants font parler « beaucoup de choses, en particulier des principes et des valeurs » (Cooren, 2010a, p. 46). Pour Cooren en effet, l'interaction est « un jeu de figures aux géométries variables » (2010a, p. 49). Nous nous intéressons à ce type de discussions, de méta-conversations, car nous rejoignons Lahire pour qui « à concentrer le regard exclusivement sur la performance, en vient à oublier les temps de préparation, d'entraînement propices à la réflexion » (1998, p. 259). C'est au contraire ce temps-là qui nous intéresse ici car « il existe toujours une réflexion pragmatiquement ancrée » (Ibid., p. 270), aussi « ce qu'il est capital d'appréhender le plus finement possible, c'est la part réflexive [...] de l'action ». 
En ce sens, notre contribution à la $\mathrm{CCO}$ se situe ici non plus seulement à un niveau théorique et empirique à travers la réflexion sur le processus de figuration et sur le concept de public, mais également à un niveau méthodologique, puisque les conversations que nous étudions peuvent être à la fois appréhendées comme des entretiens semi-directifs qui mettent en scène des " dialogues sociaux » (Magioglou, 2008, p. 64) mais aussi comme des méta-conversations en tant que " médiation et création de significations [et] co-construction de sens »(Ibid.). Si l'on retrouve à chaque fois les tours de parole successifs qui caractérisent ces modalités d'interactions, celles-ci révèlent néanmoins des modalités particulières, puisqu'elles permettent aux interlocuteurs de développer leur pensée dans une visée davantage réflexive : « il y a des idées qui semblent cristallisées alors que d'autres sont en processus d'élaboration au moment où la personne prend la parole » (Magioglou, 2008, p. 63) ; ce qui, selon nous, permet ainsi de nourrir l'analyse qui s'en trouve enrichie. Une telle démarche peut permettre de palier la critique formulée par Till Jansen (2016) à l'égard de l'École de Montréal qui, selon lui, souffre de négliger la réflexivité. À l'inverse, la nature des conversations que nous analysons permet de réintégrer la place centrale de la réflexivité.

\section{La figuration comme processus d'émergence des figures : une démarche d'objectivation sociale des expériences}

Avant d'analyser les figures du public, il convient de s'attarder sur les modalités de figuration identifiées dans les discours. Cette étape intermédiaire dans l'analyse concourt notamment à respecter les postulats méthodologiques de l'approche $\mathrm{CCO}$ qui consacrent une grande importance à la compréhension des processus (Cooren et al., 2016) en vue d'expliquer le phénomène organisationnellement constitutif de la communication. Ainsi, face à l'évanescence des publics, il est nécessaire pour les membres de l'organisation de recourir à la figuration pour engager leur point de vue dans la discussion et faire parler leur expérience des publics. Toutefois, cette figuration procède par étapes. En effet, l'analyse des conversations étudiées rend compte d'une dynamique orientée vers la recherche 
de traces, d'indices ancrés dans les expériences empiriques. Les indices, soumis au point de vue collectif, soutiennent une dynamique qui s'apparente à un effort d'objectivation, entendue ici au sens d'extériorisation des représentations dans un matériau langagier partagé. " À travers un processus d'indications mutuelles, des objets communs émergent qui ont la même signification pour un ensemble donné de personnes » (Blumer, 1969, p. 11). Cette objectivation se réalise donc par l'activité communicationnelle, qui va consister à construire la signification dans l'interaction via la mise en commun de références, via « l'intégration réciproque du point de vue de l'autre » (Madelrieux, 2016, p. 81). Le langage fonctionne ici comme "l'opérateur d'objectivation et d'instrumentalisation de la relation entre l'usage d'une chose et ses conséquences possibles » (Ibid.).

Nous montrons ainsi dans l'analyse conversationnelle des processus de figuration du caractère indiciel des objets (entendus au sens de Blumer, 1969, cf. supra) mobilisés dans les échanges. L'indice est ici appréhendé comme un « fragment arraché à la chose » (Peirce, cité par Berthelot, 2001, p. 183) : le fragment entretient un rapport de contiguïté avec la chose qu'il désigne et résulte d'un raisonnement métonymique (Ibid.). Les indices sont mobilisés pour signifier les objets : plus la signification sera collective et partagée, plus elle fera consensus, plus fort sera le degré d'objectivation de la réalité discutée. C'est donc le degré d'intégration collective des significations qui détermine le degré d'objectivation. Nous pouvons positionner le processus de figuration en fonction du degré d'objectivation comme suit :

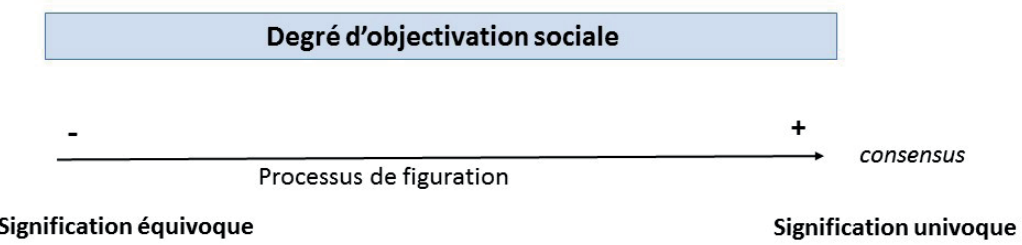

Figure 1. Degré d'objectivation sociale dans le processus de figuration 
Cela n'est pas sans faire écho à la manière dont Moscovici, dans le domaine de la psychologie sociale, entend le processus d'objectivation, qu'il articule avec le processus d'ancrage, qui tous deux, ensemble, permettent l'émergence de significations consensuelles : ils ont pour fonction de « doubler un sens par une figure, donc objectiver d'un côté... et une figure par un sens, donc ancrer de l'autre côté... les matériaux entrant dans la composition d'une représentation déterminée » $(1961$, p. 64). Molinier résume la chose ainsi : «c'est le processus d'objectivation qui produit la figure et c'est le processus d'ancrage qui lui donne sens ») (2015, p. 83). Ce dernier appelle à mobiliser un cadre de référence, nécessairement familier, pour la mise en sens.

Dans le cas qui nous intéresse, nous distinguons trois manières de procéder à la figuration pour se représenter les publics : une manière consiste à convoquer sa propre expérience individuelle, une autre consiste à faire référence à des situations d'échange qui sont apparentées à des expériences collectives, une dernière consiste à mobiliser des chiffres. Dans les deux premiers cas, lorsque par exemple les acteurs font référence au public par le recours à une expérience individuelle ou à une expérience d'échange et de mise en relation, ils considèrent " une partie » (expérience empirique localement située du public) pour le « tout » (le public tel qu'il peut potentiellement exister). Chaque fois, la relation à l'expérience empirique amène l'un ou l'autre indice. Par exemple, son propre vécu, sa propre expérience personnelle se fait vecteur de connaissance extrapolant l'expérience des publics à travers la sienne. Ainsi, ce salarié demande à une collègue de parler de son vécu pour en figurer les publics :

«Qu'est-ce que tu attendais, qu'est-ce que le public attendait? Puisque tu correspondais pleinement à ce public ».

Celle-ci se remémore alors sa propre expérience, elle se raconte comme étudiante et jeune bénévole de l'association, elle raconte le peu d'intérêt qu'elle portait pour l'information qu'elle recevait de l'association : 
«C'est plus gai d'aller voir les amis que de s'enfermer à regarder [une revue d'information] quand tu rentres chez toi un week-end par mois ».

Son récit est utilisé pour figurer les publics jeunes, sensibilisés aux enjeux de l'association sans être engagés. Elle ajoute d'ailleurs :

« J'ai jamais vraiment fait une démarche proactive pour connaitre mes publics si ce n'est que j'ai un petit avantage c'est que moi je considère, si on s'adresse par exemple au public cible des étudiants, ben je le connais intuitivement, je suis passée par cette case-là ».

Ce processus de figuration permet de matérialiser les publics grâce au recours à un discours réflexif fondé sur sa propre expérience qui se manifeste sous la forme d'une anecdote, d'un souvenir ou encore de la projection dans une situation imaginée. Cette lecture de la figuration renforce l'orientation pragmatiste qui conçoit que « nous sommes tous des participants (pratiquants) dans nos mondes sociaux. C'est à travers notre participation que nous construisons et re-construisons continûment les significations sociales qui modèlent nos pensées et nos actions 》 (Simpson, 2013, p. 159). À partir du moment où il y a tentative de verbaliser, donc de matérialiser, le propos dans l'interaction en lui conférant une signification partagée, il y a processus d'objectivation.

Une autre modalité de figuration passe par la convocation des personnes rencontrées, connues, qui deviennent représentatives des publics auxquels elles sont apparentées : l'expérience individuelle est alors apparentée à une expérience partagée. Les bénévoles, c'est-à-dire « des personnes qui s'engagent sous l'impulsion de leur "bonne volonté" et qui ne perçoivent aucune rémunération financière en contrepartie » (Vermeersch, 2004, p. 682), sont souvent mentionnés dans ce type de figuration. Ceux-ci ont l'avantage d'être identifiés, connus et reconnus par les salariés de l'association : " les bénévoles on les connaît tous », " on [les ] côtoie », " on est proche » indique ainsi une salariée. Ici la connaissance passe par la proximité et la possibilité de côtoyer les personnes. 
C'est ainsi que ces dernières deviennent « vraiment le public », comme l'exprime une salariée, voire un public idéal à l'aune duquel les autres publics vont être comparés, voire jugés. À côté des bénévoles, les décideurs politiques avec lesquels des relations privilégiées se sont créées sont également mentionnés, comme on le voit dans l'extrait suivant ${ }^{5}$ :

1 Martine : Mais je trouve que ce qui est intéressant c'est qu'on est censé

2 s'intéresser aux publics cibles et qu'on glisse sur [nom de l'organisation],

3 ce qui montre qu'on est quand même assez égocentrique qu'on n'a pas

4 grand-chose à dire de nos publics.

5 Xavier : Non, mais c'est ça oui, c'est que... il n'y a pas d'info.

6 Martine : (...) on sait pas dire grand-chose de nos publics.

7 Xavier : Oui, mais parce qu'on ne les rencontre pas souvent, et ceux

8 qu'on rencontre sont dans nos girons personnels, du coup... c'est pour toi

9 des gens, des décideurs que tu rencontres dans tes visites (...) des gens

10 qui savent que tu fais [nom de la revue] et qui viendront te dire volon-

11 tairement « ah chouette c'est bien, j'ai bien aimé », tu vois, donc on est

12 toujours influencés ou embrumés par...

\section{Extrait 1. Conversation entre salariés}

L'extrait de conversation ${ }^{6}$ ci-dessus met en lumière le processus de figuration en cela qu'il indique clairement les modalités de connaissance des publics par les salariés : pour pouvoir réellement connaître les publics, il faut les avoir rencontrés. Mais alors le processus de mise en commun n'est pas aisé puisqu'il repose sur des expériences empiriques individuelles, même si on tente de les partager et de dégager une signification commune. À l'inverse, une autre manière de figurer les publics mobilise des indices qui semblent davantage faire consensus, à savoir des chiffres, bases de données, clics. Le chiffre est un indice clé, puisqu' « en décrivant, nommant, désignant les objets pertinents à chiffrer, il les rend visibles, [...], il les fait exister » (Fauré, 2007, p. 119). Le chiffre est en effet omniprésent dans les propos des salariés. Dès le début

5 Les noms des personnes ont été modifiés pour conserver leur anonymat.

6 Au passage, notons que cet extrait montre également que, par le biais des publics, c'est bien de l'organisation dont on parle. 
de la discussion que nous sollicitons, alors qu'il ne leur est rien demandé, les salariés déclinent les revues dont ils sont responsables, qui s'adressent à des publics figurés, en indiquant à chaque fois le nombre d'exemplaires diffusés. Le nombre d'exemplaires semble s'apparenter au public, comme si par le chiffre, les salariés attribuaient un corps (Cooren, 2010c, p. 199) au public, comme on peut le saisir dans ce propos :

« Le public... le lectorat de [la revue] c'est un lectorat composé de ce qu'on appelle les décideurs, les gens qui ont une fonction qui peuvent... qu'on identifie comme ayant une fonction qui peut influer sur la décision politique et le public institutionnel, le public professionnel on va dire. C'est un public qui se retrouve dans deux axes, une diffusion papier et une diffusion via la newsletter électronique. Son... c'est 2500 exemplaires papier et la newsletter électronique est à environ 2600 envois ».

Les chiffres rythment la discussion et sont scandés tout au long de la conversation, certainement parce que " l'indication chiffrée bénéficie a priori d'un prestige incontestable : le prestige des apparences objectives, de l'évidence et du discours scientifique rationaliste » (Koren, 2009, p. 72). En ce sens, le chiffre est un indice qui fait consensus, sa signification étant a priori univoque et partagée. Ces trois processus de figuration mobilisent des indices dont la signification est plus ou moins partagée, ce qui se traduit par des degrés d'objectivation variable que nous présentons dans la figure 2 .

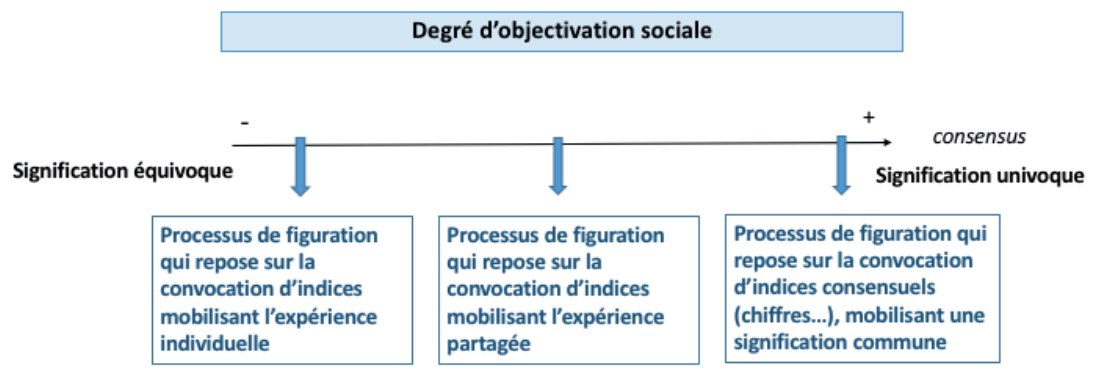

Figure 2. Variation du degré d'objectivation sociale en fonction des différents processus de figuration 
Les processus de figuration ainsi identifiés peuvent nous aider à saisir les figures dans leur qualité émergente. Plus la signification est partagée (signification univoque), plus la figure qui émerge est performative.

\section{Figures en tension : l'hybridité au cœur de l'organisation}

Deux usages dominants de la figure du public sont en tension qui émergent des processus de figuration analysés ci-dessus (voir figure 2) : une figure émane de la figuration qui repose sur la convocation d'indices mobilisant l'expérience individuelle et collective et tend à approcher le public par ses motivations et son engagement ; une autre émerge du processus de figuration qui repose sur la convocation d'indices consensuels et tend à envisager le public sous sa forme quantifiée. Chacune amène des référentiels d'évaluation divergents, que l'on peut identifier dans les extraits de conversations ci-dessous. Rappelons que celles-ci sont des extraits de discussions qui s'apparentent à des méta-conversations (Robichaud et al., 2004) dans la mesure où elles portent sur les activités et les objectifs mêmes de l'organisation envisagés à travers la question des publics.

1 Louis - Du temps où on faisait des stands...

2 Michelle - ... ceux qui signaient la pétition ...

3 Louis - Ceux qui signcnt les pétitions.

4 Martine - C'cst une porte d'entrée, d'être en contact direct avec eux

5 et on est attentif de leur offrir des portes de sortie, c'est-à-dire qu'on a

6 eu un un contact et on veut pas que ça reste un one shot, on veut gar-

7 der du lien avec eux. Ça, c'est dans l'idéal, ceux qui ont fait une vraie

8 démarche volontaire de s'abonner à des publications ou de se mettre

9 candidats bénévoles. Maintenant, ici par exemple, dans une démarche où

10 on a refait notre base de données l'année passée, on a pris la liberté nous-

11 même d'abonner des gens en se disant " tiens, c'est un profil "prof" ",

12 a priori, il serait intéressé par [nom de la revue], et barn, on abonne, on

13 abonne 1000 personnes [à la revue], à la version électronique...

14 Léo - 1000?

15 Sophie -6000 non?

16 Martine - ... en se disant, ils ont toujours la possibilité... 


\section{7 (?) - 16000 - HUUUUUM}

18 Martine - ... de se désinscrire parce que via mailchimp bon voilà, il y a

19 toujours le bouton pour se désinscrire... C'est toujours en version élec-

20 tronique qu'on prend cette liberté-là, on le fait pas sur la version papier.

21 (chuchotements)

22 Martine - ... parce que là, c'est quand même plus insidieux, plus difficile

23 de faire une démarche de désinscrire. Donc voilà, ça c'est un peu... par

24 contre là, et ben c'est ce qui peut expliquer aussi que sur euh des... parce

25 qu'on a pas de retour sur le papier, donc on ne sait pas, nos publics, sur

26 le papier, on ne sait pas ce qu'ils en font mais sur l'électronique on sait

27 ce qu'ils en font et le taux d'ouverture et de clics... mis à part André, qui

28 a quand même un taux de clic qui est pas trop mauvais, je vais dire qu'il

29 est faible quant au pro... par rapport à ce qu'on met comme énergie et

30 comme argent là-dedans, il est très faible.

31 Léo - Mais parfois vous avez des retours je dirais ultra qualitatifs, donc

32 par exemple sur le travail plaidoyer-décideurs il est clair et net que dans

33 le fichier on décide nous-mêmes tiens, tel parlementaire suit nos pro-

34 blématiques ou tel fonctionnaire dans l'administration de la coopéra-

35 tion au développement il suit nos thématiques, donc on va leur envoyer

36 nos publications et parfois on a des retours ultra qualitatifs, c'est-à-dire

37 qu'on entend par hasard que tel politicien à la commission ou au par-

38 lement a vraiment beaucoup aimé tel article ou telle publication et que

39 ça l'a influencé. C'est archi qualitatif, parce que ça arrive... une fois par

40 an?

41 Louis - Non, quand même.

42 Léo - deux, trois?

43 Louis - Non, plus.

44 Léo - Plus. Allez, ok. C'est lui qui le dit.

45 (rires)

46 Louis - Non mais c'est vrai.

\section{Extrait 2. Conversation entre salariés}

On voit dans cet extrait deux figures principales animer la discussion : celle du public citoyen qui est mentionnée au tout début (lignes 1 à 8 ) en évoquant la démarche de signer des pétitions. Le public citoyen est présenté comme le public idéal pour cette démarche volontaire qui est la sienne, qui s'apparente à celle du don et de l'engagement. Le public citoyen apparaît également un 
peu plus tard, dans la dernière partie de l'extrait (lignes 31 à 38), quand il s'agit de qualifier « des retours ultra qualitatifs » qu'un salarié, Léo, a reçu de l'un ou l'autre responsable politique. Ces derniers sont en effet également envisagés comme des publics de l'organisation et la proximité qui apparait ici permet de les qualifier également de public citoyen.

À l'opposé, la figure du public client anime également la discussion. Ici, le public ne vient pas à l'organisation, c'est l'organisation qui vient à lui (lignes 10 à 13). Il devient public à « marche forcée ", voire à son insu, dans une démarche imposée, par l'incorporation dans une base de données. Pour ce faire, l'organisation " cible » les profils idoines, comme celle du professeur par exemple, qui se trouve ainsi abonné à une revue sans en avoir fait la demande. La manière de qualifier ce public n'est pas ici la qualité du lien - d'autant plus que celui-ci apparait peu fort, pour ne pas dire fragile - mais la mesure par le chiffre (lignes 14 à 17). On constate d'ailleurs l'utilisation d'un vocabulaire gestionnaire : le « taux » (d'ouverture et de clic), relié ici à la notion de rendement (puisqu'on évalue le taux par rapport à l'énergie et à l'argent mobilisés). Le public client est ainsi matérialisé par les bases de données, les chiffres (nombre de clics, d'envois de publications, etc.), comme en témoignent ces extraits d'entretiens semi-directifs avec les salariés. Léo par exemple explique :

«On est plus qu'avant dans les statistiques et des vérifications de statistiques et des préoccupations sur des clics [...]. Tu vois sur internet le nombre de gens qui cliquent, et ça, ça booste tout le temps les discussions sur les publics cibles ».

Xavier note quant à lui que « le seul moyen de mesurer l'impact ou le résultat [d'une revue] c'est de mesurer la fréquentation électronique du contenu $»$.

À l'inverse, le public envisagé comme citoyen se constitue à propos d'un enjeu public. Il est figuré essentiellement par la convocation des bénévoles, qui n'est d'ailleurs pas quantifiée. Peut-être parce que les chiffres obtenus seraient alors trop faibles. Peut-être aussi parce que la figure du citoyen est justement aux antipodes 
de celle du client : c'est ici la qualité de la relation qui prime, comme l'explique un salarié à l'occasion d'un entretien semidirectif. Selon lui si les bénévoles restent c'est justement parce que :

« On n'est pas un numéro. [...] On reste parce qu'on n'est pas un numéro, $[\ldots]$ on a l'impression d'être partie prenante de quelque chose. On ne se sent pas instrumentalisé ».

Notons d'ailleurs l'utilisation du « on » qui renvoie ici encore à un processus de figuration reposant sur une expérience partagée. La figure d'un public idéal émerge ainsi dans la description qui est donnée des bénévoles "qui sont dans une démarche volontaire d'apprendre plus », selon un salarié. Cette représentation des bénévoles n'est pas sans évoquer l'idéal du public politique, caractérisé par une « compréhension commune de ce qui est fait ensemble », « une manière déterminée d'agir et d'agir ensemble », « un acte commun de focalisation » (Quéré, 2003, p. 121). Il n'est pas non plus sans faire écho à l'idéal d'un public actif et participatif à la Dewey (1927/2010), les individus le constituant se sentant liés les uns aux autres dans un horizon de vie collective. Un salarié résume ainsi cette figure du public politique :

« On a une vision du citoyen dans son rôle politique et non dans son rôle de consommateur. On veut les conscientiser aux enjeux politiques du monde $»$.

On retrouve ces deux figures antagonistes dans l'extrait suivant :

1 Xavier - Pour moi c'est fondamenlal qu'une maison d'édition quelle

2 qu'elle soit, soit force de proposition de contenu.

3 Martine - Oui, je dis pas, mais...

4 Xavier - C'est notre plus-value, parce que la dérive c'est...

5 Michelle - faire que ce que le public veut...

6 Xavier - c'est... RTL -TVI c'est... Plug TV quoi.

7 Martine - Oui, non 
8 André - C'est la DH.

9 Martine - Non, non... ne pas faire, je voulais pas que ce soit ça comme

10 message qui passe. Ce que je voulais dire c'est que quelque part il y

11 a moyen de rester intègre, enfin, franchement, je trouve que c'est très

12 intéressant, entre rester intègre, qui on est, en adéquation avec notre

13 identité, voilà, on va pas essayer de vendre du people parce que les

14 gens ont envie de lire du people, nous on a un série de messages à

15 livrer, mais par contre, sur la palette de possibles, il y a des choses qui

16 collent mieux à l'attente de nos publics. Et ça, cet entre-deux, ce déno-

17 minateur commun, si on connaissait mieux notre public on y collerait

18 mieux. Ça veut pas dire évidemment d'aller dire des choses qui ne sont

19 pas de nous et qui ne collent pas avec qui on est hein.

20 Xavier - Oui, entre le choix de 3 sujets, il y a peut-être un sujet qui

21 parlera mieux que l'autre et qui sera plus heu... trendy. Mais sur le fond

22 et sur le message qu'il faut faire passer, on doit être très cohérents et

23 intransigeants. C'est à nous à faire un plaidoyer et à proposer une poli-

24 tique de plaidoyer. On fait de l'éducation, c'est très prétentieux hein

25 de le dire mais on fait de l'éducation au développement. Euh, voilà. Le

26 prof, il ne demande pas à ces élèves « bon les gars qu'est-ce que vous

27 voulez voir aujourd'hui »?

28 Sophie - On....

99 Xavier - Enfin, si dans certaines écoles ça se passe...

30 Sophie - Oui, si...

31 Xavier - Oui, mais bon.

32 Martine - Mais ça donne des bons ré... Je pense qu'il y a une logique,

33 en tout cas je trouvais intéressant, on a eu un temps de réflexion en

34 début d'année, un peu en dehors des sentiers battus ici dans les bureaux

35 et on disait, c'est vrai qu'avec nos publics on est très top-down, là je

36 parle pas juste publications, mais est-ce qu'on devrait pas être dans une

37 démarche plus intégrée et c'est vrai que dans l'exemple de la campagne

38 on se disait plus s'associer déjà aussi avec des associations et pas juste

39 pour les instrumentaliser après en disant « relayer ce qu' on veut faire »

40 mais en réfléchissant nous et même chose, par exemple [nom de la

41 revue...] pourrait être une revue peut-être un peu plus ouverte, collec-

42 tive, avec d'autres assos qui auraient peut-être les mêmes objectifs que

43 nous, ou... pour mieux les valoriser.

44 Michelle - Mais moi je trouve que quand même [nom de la revue], 45 le fait qu'on y associe des bénévoles dans la réflexion des thèmes, la

46 construction des articles, ça montre que c'est quand même une démarche 
44 intégrée tu vois. On choisit les articles, on choisit les thèmes avec eux,

45 alors après ils font des propositions, on a un droit deregard nous aussi

46 parce qu'on sait très bien que nos publics ils aiment bien entendre par-

47 ler d'agriculture au Nord et de consommation d'alimentation durable et

48 que nous on a quand même les messages qu'on veut faire passer qui ne

49 ont pas nécessairement ceux-là, mais, je trouve que c'est quand même

50 une belle démarche participative quoi.

51 Martine - Oui, sur une publication sur six.

52 Michelle - Oui, oui, oui, mais il y en a une quoi.

53 Sophie - Et qui est celle qui touche un public, tu vois, le plus varié..., 54 le plus...

55 Louis - Le plus large...

\section{Extrait 3. Conversation entre salariés}

Cet extrait permet d'enrichir l'analyse, puisqu'il reprend les deux figures antagonistes du public qui se matérialisent dans et à travers les interactions (Cooren, 2012), en les confrontant à la construction identitaire de l'organisation. On voit en effet dès le tout début de l'extrait (lignes 1 à 2) que celle-ci s'assimile à une maison d'édition, ce qui peut paraître surprenant pour une ONG d'aide au développement. Une telle expression souligne le rôle central qu'elle donne aux publications qu'elle édite : parce que l'éducation au développement passe par ces revues, celles-ci sont au centre de la réflexion sur les publics. Ce que montre également cet extrait c'est que parler des publics, c'est, pour les salariés, parler d'eux-mêmes et parler de l'organisation ${ }^{7}$. On comprend alors aisément en quoi les figures des publics sont performatives et transportent avec elles des valeurs et des principes. Faire parler les publics via les figures, c'est faire parler sa vision de l'organisation. On voit réapparaître la figure du public client envisagé ici dans une approche médiatique : c'est celui à qui on donne des choses de basse qualité à voir ${ }^{8}$ pour lui plaire, pour « brosser dans le sens du poil » (lignes 5 à 8).

7 Une indication supplémentaire que l'on est ici en présence d'une méta-conversation.

8 RTL-TV, Plug-TV, DH sont des médias belges populaires, qui arrivent souvent en tête de classement en terme de part d'audience mais dont la qualité ne fait pas l'unanimité. 
À l'inverse, le public citoyen, qui apparaît dans la deuxième partie de l'extrait, est celui qui s'intéresse, qui veut être associé, voire qui veut être éduqué. L'organisation semble osciller entre les deux. Cela apparaît assez clairement quand Martine parle de « l'entre-deux » (ligne 16) dans lequel l'organisation se trouve, cette nécessité de trouver l'équilibre pour « rester intègre » (ligne 11), entre l'objectif d'attirer et de plaire à un public large, et de l'autre « proposer une politique de plaidoyer » (ligne 23). On saisit aussi dans cet extrait l'importance de la question des publics pour l'organisation, celle-ci ayant été déjà évoquée quelques mois avant (lignes 33-34) : le public devient le texte auquel il est fait référence dans la conversation. On comprend enfin le défi qui se pose à l'organisation dans la manière d'animer le public citoyen, que l'on veut faire participer, certes, tout en gardant le contrôle de la relation et du contenu produit.

Cet extrait, comme l'autre, dévoile des figures en tension, entre celle du public client et celle du public citoyen. Ramenées à l'identité de l'organisation, la première peut évoquer l'éthique de responsabilité, la deuxième l'éthique de conviction, telle que décrites par Weber".

Quand le public client est celui de la performance par la mesure du chiffre, le public citoyen est celui de l'engagement par la mesure de la qualité du lien. Ces différentes figures entrent en contradiction car elles ne racontent pas les mêmes choses des publics et, par conséquent, de l'organisation : d'un côté l'objectif est de toucher le maximum de personnes de manière à pouvoir démontrer la force de frappe quantitative de l'association et asseoir ainsi sa légitimité, de l'autre l'objectif est d'expliquer, échanger et éduquer pour permettre la prise de conscience citoyenne et favoriser la mobilisation.

Dans cette tension entre ces deux figures antagonistes transigent des effets de valeurs contradictoires (Gagné, 2011), d'ordre davantage économique et marchand pour la figure du public client et davantage moral et civique pour la figure du public citoyen. La

9 Le partisan de l'éthique de conviction est animé par l'obligation morale, le partisan de l'éthique de responsabilité par une rationalité instrumentale fondée sur la calculabilité (Fleury, 2009). 
première fait de la performance le critère ultime, la seconde fait de l'engagement la valeur dominante.

\begin{tabular}{|l|l|}
\hline \multicolumn{1}{|c|}{ Figures textualisées du public } & \multicolumn{1}{|c|}{ Valeurs } \\
\hline Le public client & Performance \\
& Distance \\
& Mesure \\
& Gestion \\
\hline Le public citoyen & Engagement \\
& Proximité \\
& Echange \\
& Démocratie \\
\hline
\end{tabular}

Figure 3. Correspondance entre figures et valeurs

Si les deux figures qui émergent du discours existent bien par leur incarnation communicationnelle (Cooren, 2012), elles démontrent également leur caractère performatif ou agentif par leur capacité à pointer vers des orientations d'action pour l'organisation. L'effet pratique (Taylor et Robichaud, 2004, p. 401) de ces figures ${ }^{10}$, autrement dit la " différence » (Cooren, 2010a, p. 39) qu'elles introduisent, est une « co-orientation » (Taylor et Robichaud, 2004, p. 401) des acteurs autour de l'objet du public ; objet autour duquel l'activité même de l'organisation se structure. Ainsi, par exemple parler du public client, c'est faire de l'organisation une organisation performante.

Si les deux figures " organisent l'organisation », il apparait que la première, mesurée par des indicateurs quantitatifs et incarnée dans des chiffres, est celle qui fait davantage autorité. On la retrouve en effet partout, et même quand la figure du public citoyen apparaît, elle est revisitée, recadrée, à l'aune des chiffres et de la quantification. Autrement dit, même quand le public est approché par la qualité de la relation, il y a automatiquement tentative de quantifier celle-ci (comme on peut le saisir à la fin de l'extrait présenté plus haut). La performance mesurée par le chiffre reste donc centrale dans l'animation des figures, où l'on voit ainsi

10 Cooren (2012) utilise les termes « figure » et « agent » de manière interchangeable. 
l'une prendre le dessus sur l'autre. Cette quantification du public est reprise dans le rapport d'activité, que l'on peut appréhender ici comme une forme de stabilisateur des conversations (Taylor, 1993), mais aussi en tant «qu'inscription, prescription et sanction » (Jolivet \& Vasquez, 2011, para. 30) qui dispose par conséquent d'une forte agentivité. $\mathrm{Si}$ « le texte fournit au groupe un moyen d'interprétation de ses actions et de leurs significations » (Taylor, 1993, para. 111), on voit là encore la place centrale du chiffre que mobilise la figure du public client.

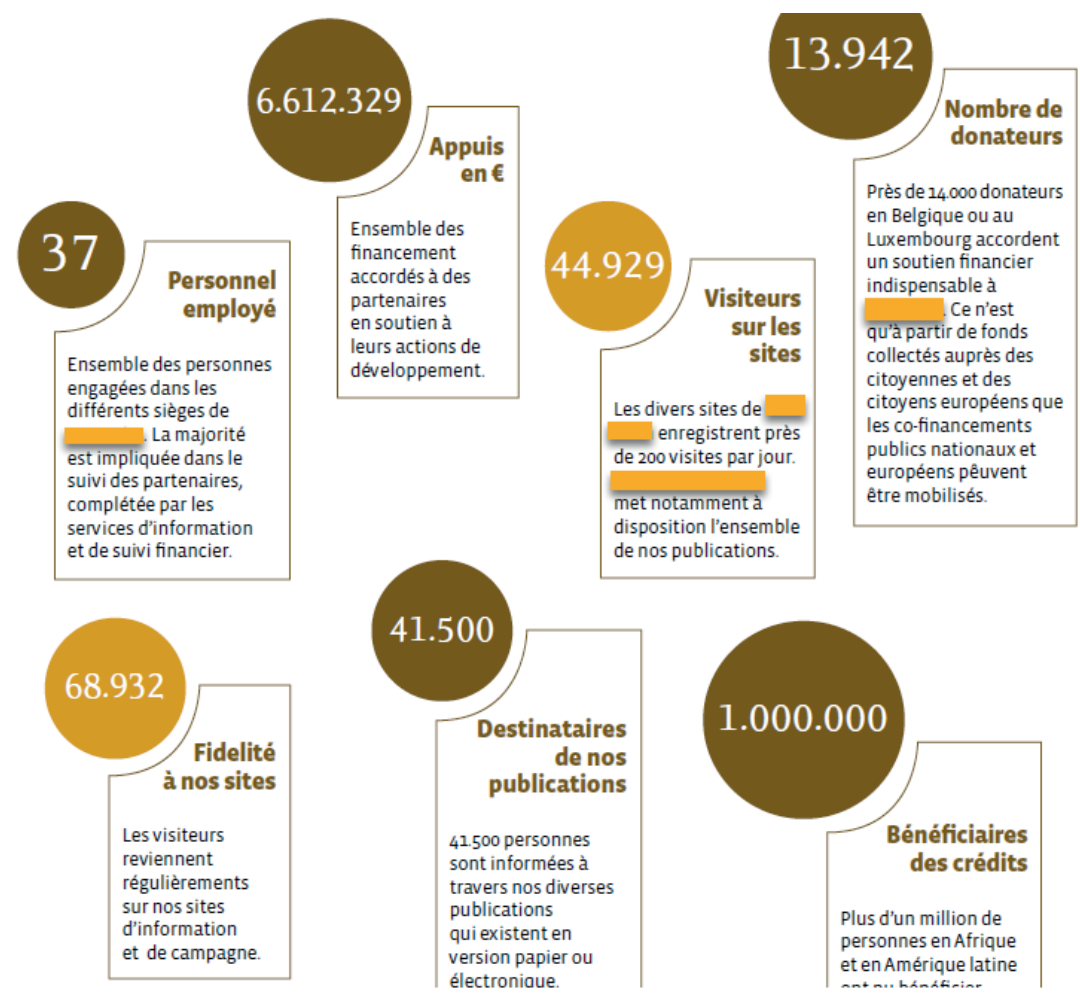

Extrait 4. Rapport d'activité (2013)

Cooren rappelle que le monde qui nous entoure est rempli "d'acteurs aux ontologies variables qui s'entre influencent les uns les autres » (2010a, p. 38). Le chiffre peut ainsi être appréhendé comme l'un d'entre eux, stabilisé par le texte, et l'on a vu, 
« renforcé par la conversation, qui lui confère une légitimité certaine, par le fait même d'être respectivement interpellé » (Taylor, 1993, para. 111). En cela, la figure du public client semble faire autorité, mais il ne faudrait pas pour autant croire que la figure du public citoyen disparait complètement. Cette dernière est mobilisée quand il s'agit notamment d'apprécier l'engagement des bénévoles dans l'organisation. Autrement dit, derrière l'acception « public » se cachent deux figures dont l'autorité dépend des configurations mises en jeu. Comme pour le patient à l'hôpital (Jolivet \& Vasquez, 2011), ici aussi la pluralité des figures du public représente un défi pour l'organisation qui cherche à les concilier, et doit pour cela appréhender des logiques contradictoires, que l'on peut résumer ainsi : celle de la performance et du nombre, celle de l'engagement et de la relation. Par conséquent, on voit bien que les figures mobilisées amènent avec elles des formes organisationnelles antagonistes mais articulées, voire entremêlées, et participent en cela à la construction de l'organisation.

\section{Conclusion}

L'entrée par les publics nous permet d'approcher les tensions qui agitent et constituent l'organisation. Derrière l'acception du terme " public », différentes figures sont en effet en compétition, qui émergent de processus de figuration au degré d'objectivation sociale variable. Chaque figure propose une vision et des qualifications différentes, parfois contradictoires, comme c'est le cas de la figure du public client et celle du public citoyen. La première émerge d'un processus de figuration qui convoque des indices qui font consensus, comme les chiffres, la seconde d'un processus de figuration au degré d'objectivation sociale moindre parce qu'il repose sur des expériences empiriques à la signification davantage équivoque : la mise en sens dans l'interaction se joue différemment dans chacun des processus et cela n'est pas sans conséquence sur la figure qui émerge. On peut ainsi se demander si le fort degré d'objectivation sociale qui caractérise le processus de figuration duquel la figure du client émerge n'est pas sans lien avec sa domination. Dit autrement, de par sa capacité à générer plus aisément 
le consensus et donc une co-orientation des acteurs, cette figure semble démontrer une plus grande force d'agentivité.

Ces remarques témoignent de la nécessité, pour la $\mathrm{CCO}$, de penser à des instruments méthodologiques qui permettent d'avoir accès à la réflexivité des acteurs puisqu'elle est mobilisée dans l'action de figuration. Accéder à la réflexivité des acteurs, c'est comprendre la manière dont ceux-ci font sens de leur situation, en évitant ainsi le risque de sur-interpréter leurs actions à partir du sens que le chercheur, seul, attribue à celles-ci. Il convient par conséquent de réintégrer dans l'analyse la notion de réflexivité, à la fois du côté du chercheur et du côté des acteurs étudiés, car « il existe toujours une réflexion pragmatiquement ancrée, indissociable de l'action en cours et des éléments du contexte immédiat » (Lahire, 1998, p. 270). Pour accéder à celle-ci, différents outils empiriques peuvent être mobilisés, comme des entretiens individuels et collectifs. L'objectif ici est de passer de l'implicite des interactions à l'explicite de l'explication de celles-ci par les acteurs eux-mêmes. Ce qui nous permet d'accéder ainsi au processus d'ancrage, de mise en sens, d'action de figurer : celui-ci s'ancre en effet dans des cadres de référence qu'on ne voit pas nécessairement apparaître, ou qu'on ne reconnaît pas (parce qu'on ne les connaît pas) dans l'interaction; il faut donc aller au-delà de l'interaction locale pour pouvoir les représenter et être en capacité d'identifier le sens que leur donnent les acteurs.

Théoriquement, l'approche par monde social ${ }^{11}$ peut apparaître heuristique en cela qu'elle permet de contextualiser, terme qui désigne dans sa racine latine « l'action de tisser ensemble, de relier ou de former par assemblage » (Lahire, 2012, p. 229). Ainsi, en s'intéressant aux « univers de discours » (Strauss, 1978), 1'approche par monde social permet de donner sens aux discours des acteurs, aux procédés de figuration, puisque ceux-ci mobilisent des indices qui se réfèrent à ces univers. De la même manière, elle permet de rendre plus clairement compte des inférences que fait le chercheur quand il étudie les interactions : comment en

11 On entend par monde social « un univers de réponses mutuelles et régularisées » (Shibutani, 1955, p. 566) ; autrement dit un « univers de discours » (Strauss, 1978, p. 120) entendu comme un espace discursif profondément relationnel. 
effet reconnaître les figures si ce n'est en faisant appel à la fois à la signification des acteurs étudiés et aux connaissances tacites que le chercheur a été puiser dans son appréhension des mondes sociaux?

L'approche par monde social permet, dans le cas qui nous intéresse, d'observer - à travers le rapport de force entre deux figures antagonistes du public - la pénétration du monde marchand, et plus particulièrement du monde du marketing, dans une association à but non lucratif ${ }^{12}$. Dans le monde du marketing, comme le soulignent Floris \& Ledun, « tout échange social tend à être perçu comme un échange quantifiable entre un fournisseur et un client », dans lequel « la figure du client est [...] le noyau » (Floris \& Ledun, 2005, para. 21). Marion (2004) rappelle également que les notions d'audience, de segmentation, de cible sont centrales dans les conceptualisations du marketing. On perçoit dans les conversations analysées comment ces notions, tributaires d'une conception marketing, sont transportées dans les manières de figurer les publics et agissent par conséquent sur la signification même de l'organisation telle qu'elle est partagée par ses membres. On les retrouve dans les conversations analysées dans cette recherche ; ce qui semble confirmer le constat de Sanders (2012) qui estime que la logique du marché a pénétré les organisations à but non lucratif. Sanders invite à s'intéresser aux tensions et contradictions que cette logique amène ; tensions et contradictions qui d'ailleurs sont le signe, selon lui, de la nature dialectique du processus organisant. Autrement dit, la tension organisante (organizing tension) est au centre de l'activité non lucrative. L'organisation apparaît ainsi comme " un champ de tensions sans frontières nettes, où différentes rationalités et discours coexistent et s'entrecroisent » (Ever, 2000, p. 569).

12 Rappelons en effet que non seulement les frontières des mondes sociaux sont plus ou moins poreuses et perméables, mais elles sont également mouvantes. 


\section{Références}

Abbott, A. (2016). La pertinence actuelle de l'école de Chicago. Dans Demazière, D. \& Jouvenet, M. (dir.). Andrew Abbott et l'héritage de l'école de Chicago. Volume 1 (En temps et lieux), Paris : EHESS.

Assogba, H., Coutant, A., Domenger, J. C. \& Latzko-Toth, G. (2015). Les publics imaginés et réels des professionnels d'internet, Communication 33(2). Disponible à : https://journals.openedition.org/communication/5704

Baudry, G. (2016). Du local au global : agentivité et dynamiques représentationnelles d'organisation. Le cas d'une entreprise internationale du secteur de l'aéronautique et du spatial. Thèse de doctorat, Université de Toulouse.

Berthelot, J.-M. (2001). Épistémologie des sciences sociales. Paris : PUF.

Blumer, H. (1969). Symbolic interactionism : perspective and method. NJ : Prentice Hall Englewoods Cliffs.

Carion, F. (2010). La communication associative. À la recherche d'un équilibre entre logique fonctionnelle et logique relationnelle ? Communication information médias théories pratiques 28(1), 193-206.

Cefaï, D. (2015). Mondes sociaux. Enquête sur un héritage de l'écologie humaine à Chicago. SociologieS. Disponible à : http://journals.openedition.org/sociologies/4921.

Cooren, F. (2010a). Ventriloquie, performativité, et communication. Ou comment faiton parler choses? , Réseaux 5(163), 33-54.

Cooren, F. (2010b). Comment les textes écrivent l'organisation. Figures, ventriloquie et incarnation, Études de communication 34, 23-40.

Cooren, F. (2010c). Action and agency in dialogue : passion, ventriloquism and incarnation. Amsterdam : John Benjamins.

Cooren, F. (2012). Communication theory at the center: Ventriloquism and the communicative constitution of reality. Journal of Communication 62(1), 1-20.

Cooren, F. (2013). Manières de Faire Parler : Interaction et Ventriloque, Latresne : Le Bord de l'eau.

Cooren, F. \& Martine, T. (2016). Matérialité, communication et organisation : La vidéo-filature d'une idée, Revue française des sciences de l'information et de la communication (9). Disponible à : http://journals.openedition.org/rfsic/2065

Cooren, F., Bartels, G. \& Martine, T. (2016). « Organizational communication as process ». Dans Langley, A. \& Tsoukas, H. (dir.). The Sage Handbook of Process Organization Studies (p. 513-527), Sage.

Dayan, D. (1992). Les mystères de la réception, Le Débat 71(4), 141-157.

Denis, J. (2008). Projeter le marché dans l'activité. Les saisies du public dans un service de production télévisuelle, Revue française de socio-économie 2(2), 161-180.

Dewey, J. (1927/2010). Le public et ses problèmes, Paris : Folio.

Errecart, A. (2013). Des organisations en partenariat : un espace communicationnel hybride au prisme de l'analyse de discours, Sciences de la société 88, 100-116.

Esquenazi, J.-P. (2006). Les médias et leurs publics. Dans Olivesi, S. (dir.). Sciences de l'information et de la communication (p. 11-26). Grenoble : PUG.

Esquenazi, J.-P. (2009). Sociologie des publics. Paris : La Découverte.

Ever, A. (2000). Les dimensions sociopolitiques du tiers secteur. Les contributions théoriques européennes sur la protection sociale et l'économie plurielles, Sociologie $d u$ travail 42(4), 567-585. 
Fauré, B. (2007). Une analyse communicationnelle des outils de gestion et de contrôle. Quels apports pour les recherches en communications organisationnelles, Сотmunication et organisation 31, 109-123.

Fleury, L. (2009). Max Weber. Paris : PUF.

Floris, B. \& Ledun, M. (2005). Le marketing, technologie politique et forme symbolique du contrôle social, Études de communication 28. Disponible à : https://journals.openedition.org/edc/303

Gagné, P. (2011). Note de lecture. COMMposite 14(1), 84-94. Disponible à : http:// www.commposite.org/index.php/revue/article/viewFile/31/31

Jansen, T. (2016). Who is talking ? Some remarks on nonhuman agency in communication, Communication Theory 26(3), 255-272.

Jojczyk, J., Lambotte, F., Mnasri, S. \& Wathelet, E. (2016). Les articulations des textes et des conversations dans l'approche de la communication constitutive des organisations, Revue française des sciences de l'information et de la communication 9. Disponible à : https://journals.openedition.org/rfsic/2197

Jolivet, A. \& Vasquez, C. (2011). Reconfiguration de l'organisation : suivre à la trace des figures textualisées - le cas de la figure du patient, Études de communication 36, 129-146.

Koren, R. (2009). Le récit de chiffres : enjeux argumentatifs de la "narrativisation" des chiffres dans un corpus de presse écrite contemporain, A Contrario 1(12), 66-84.

Kruckeberg, D. \& Vujnovic, M. (2010). The death of the concept of publics (plural) in 21 st century public relations, International Journal of Strategic Communication 4(2), 117-125.

Lahire, B. (1998). L'homme pluriel. Les ressorts de l'action. Paris : Nathan.

Lahire, B. (2012). Monde pluriel. Penser l'unité des sciences sociales, Paris : Seuil.

Latour, B. (2005). Changer de société, refaire de la sociologie. Paris : La Découverte.

Livingstone, S. (2004). Du rapport entre audiences et publics, Réseaux 4(126), 17-55.

Madelrieux, S. (2016). La philosophie de John Dewey. Paris : Vrin.

Magioglou, T. (2008). L'entretien non directif comme modèle générique d'interactions, Les Cahiers Internationaux de Psychologie Sociale 2(78), 51-65.

Marion, G. (2004). Idéologie marketing. Paris : Éditions d'organisation.

Meunier, J. P. (2003). Le problème de la représentation mentale : représentation propositionnelle et/ou représentation imagée, Recherches en communication 19(19), 103-112.

Moliner, P. (2015). Objectivation et ancrage du message iconique. Propositions théoriques et pistes de recherche, Sociétés 4(130), 81-94.

Moscovici, S. (1961). La psychanalyse, son image, son public. Paris : PUF.

Quéré, L. (2003). Le public comme forme et modalités d'expérience, Dans D. Cefaï \& D. Pasquier (dir.). Les sens du public. (p. 113-134). Paris : PUF.

Robichaud, D., Giroux, H. \& Taylor, J. R. (2004). The meta-conversation : the recursive property of language as the key to organizing, Academy of Management Review 29(4), 617-634.

Sanders, M. L. (2012). Theorizing nonprofit organizations as contradictory enterprises: understanding the inherent tensions of nonprofit marketization, Management Communication Quaterly 26(1), 179-185.

Shibutani, T. (1955). Reference groups as perspectives, American Journal of Sociology $60,522-529$. 
Simpson, B. (2013). Le pragmatisme, Mead et le tournant pratique, Activités 10, 10-11. Strauss, A. L. (1978). A Social World Perspective. Dans Denzin N. (dir). Studies in Symbolic Interaction, 1 (p. 119-128). Greenwich, CT : JAI Press.

Strauss, A. L. (1982) Social Worlds and Legitimation Processes. Dans Denzin, N. (dir.). Studies in Symbolic Interaction, 4, (p. 171-190). Greenwich, CT : JAI Press.

Taylor, J. R. (1993). La dynamique de changement organisationnel. Une théorie conversation /texte de la communication et ses implications, Communication \& Organisation 3. Disponible à : https://journals.openedition.org/communicationorganisation/1619

Taylor, J. R. \& Robichaud, D. (2004). Finding the organization in the communication: Discourse as action and sensemaking. Organization 11(3), 395-413.

Trethewey, G. E. A. \& Ashcraft, K. L. (2004). Special issue introduction: Practicing disorganization: The development of applied perspectives on living with tension, Journal of Applied Communication Research 32(2), 81-88.

Vermeersch, S. (2004). Entre individualisation et participation : l'engagement associatif bénévole, Revue française de sociologie 4(45), 681-710.

\section{(9) $(\Theta \Theta \Theta$}

«Attribution - Pas d'Utilisation Commerciale - Pas de Modification 4.0 International» (CC BY-NC-ND) 